\title{
Heat transfer problem analysis in three dimension tromol brake system problem
}

\author{
Tulus Tulus*, Sudirman Sudirman, U. Sinulingga, and T.J. Marpaung \\ Universitas Sumatera Utara, Department of Mathematics, Padang Bulan 20155 Medan, Indonesia
}

\begin{abstract}
One of the physical problems that can be found in heat transfer is heat transfer to a vehicle brake. In this study a focused vehicle is a motorcycle. Where the heat transfer of the brake system on the motorcycle in this study was completed finite element method. The purpose of this study is to analyse one of the problems of displacement in the three dimensions of the motorcycle brake. So, the results obtained in the form of the best time in the braking for motorcycles. The settlement method is to use one of the partial differential equations for heat transfer. One of them is the Elemenisation model is the braking system. As is known braking system is a system used to slow and stop the vehicle. During the braking phase, the vehicle's kinetic energy will be converted into heat energy due to the friction action of the brake system. The heat flux produced by effects to the brake lining is ideally dissipated into the environment to avoid brake friction pad emissions. The heat produced has a tendency to evaporate the brake fluid and the disc brake surfaces will become hot. Based on the results obtained, when the braking system is in operation, the most significant heat transfer in $t$ $=3.8 \mathrm{~s}$. This time is the best time to avoid excessive brake fluid evaporation and the rapid breakdown of the brake surface due to excessive heat.
\end{abstract}

\section{Introduction}

Heat transfer is one of the many physical problems to be found. Further analysis in any matter related to heat transfer is a very important study. Where from the analysis performed can solve a problem of heat transfer or at least reduce the negative impact in heat transfer. So, from the study obtained optimal results. In this research the problem in heat transfer that will be discussed is the heat transfer in the tromol brake of a motor vehicle. And the analysis and simulation are done in three-dimensional form.

As is known, the increasing number of motorcycles is very drastic at this time, making the brakes become a vital component for safety, especially in brake lining. That is because the brake system on the brake motorcycle has important reasons to be considered. Braking failure can be caused by several things, namely, overheating disc brakes or damage to the brake lining. Where brake lining is a very important component of a motorcycle because it works to slow down and stop the rotation of the shaft, controlling the shaft and for the safety of the rider. When the brakes are used, the brakes and tromol boards experience heat transfer and friction.

The process of heat transfer during braking can cause braking failure. Heat transfer is the process of moving heat or thermal energy due to the temperature difference. Where, the heat energy will move from higher medium temperature to lower medium temperature [1]. The heat transfer process can occur with convection, conduction, and radiation [2]. The heat transfer process will continue until there is a temperature equilibrium occurring in both mediums. After the temperature has been balanced, we will analyse the relationship of each factor that influences the temperature obtained (speed, acceleration, etc.) with the length of time in the process of this research.

\section{Method}

\subsection{Brake}

Brake is a device use to slow or stop the movement of wheel, automatically the movement of vehicles become slow. The kinetic energy thrown away from the movement of this devices is usually transformed as heat because friction. Generally, brake can be defined as a device that is useful to slow or even to stop the movement of wheel. Brake system can be described into two types, disc brake and tromol brake [3].

\subsection{Heat Transfer}

Heat transfer is the process of heat energy movement because of the difference in temperature. Heat energy will move from higher medium temperature to lower medium temperature. There are three mechanisms at heat transfer they are conduction, convection, and radiation [4].

\footnotetext{
* Corresponding author: tulus@usu.ac.id
} 


\subsection{Partial Differential Equation}

A partial differential equation is an equation that involves more than one independent variables, an unknown function as a dependent variable. The partial derivatives of the dependent variables with respect to the independent variables [1].

Generally, partial differential equation can be write as,

$$
F\left(x_{1}, x_{2}, \cdots, x_{n}, \frac{\partial y}{\partial x_{1}}, \frac{\partial y}{\partial x_{2}}, \cdots, \frac{\partial y}{\partial x_{n}}\right)
$$

where, $y\left(x_{1}, x_{2}, \cdots, x_{n}\right)$ is unknown function and $F$ is given function. And generally, formula for heat transfer at finite element method use partial differential equation i.e.,

$$
p c \frac{\partial T(x, t)}{\partial t}=k \frac{\partial^{2} T(x, t)}{\partial t}
$$

where $T(x, t)$ is temperature at $x$ location and at $t$ time.

\subsection{Weak Formulation}

From two-point boundary value problem,

$$
\begin{gathered}
-\frac{\partial^{2} u}{\partial x^{2}}=f \quad \text { in }(0,1) \\
u(0)=0 \\
u^{i}(0)=1
\end{gathered}
$$

if $u$ is solution and $v$ is any function where $v(0)=0$, integration by parts yields,

$$
\begin{array}{r}
(f, v)=\int_{0}^{1} f(x) \\
v(x) \partial x=\int_{0}^{1} u^{\prime \prime}(x) v(x) \partial x \\
=\int_{0}^{1} u^{\prime}(x) v^{\prime}(x) \partial x=a(u, v)
\end{array}
$$

obtained,

$$
\left.V=v \in L^{2}(0,1): a(v, v)<\infty \text { and } v(0)=0\right\}
$$

then the solution can characterize by,

$$
a(u, v)=(f, v) \forall u, v \in V
$$

which is called the variational or weak formulation.

\subsection{Ritz-Galerkin Method}

If $S \subset V$ be any (finite dimensional) subspace, then the equation from weak formulation with $V$ replaced by $\mathrm{S}$, namely

$$
\left(u_{s}, v\right)=(f, v) \quad \forall u, u_{s} \in S
$$

can make easy for find solve from weak formulation because the function in a discrete form and this is called the Ritz-Galerkin Method.

\subsection{Error Estimates}

For observing the fundamental orthogonality relation $u$ and $u_{s}$. Taken equation from Ritz-Galerkin Approximation and weak formula implies,

$$
\left(a-u_{s}, w\right)=0, \quad \forall w \in S
$$

this equation and its variation we get from Ritz-Galerkin method. Now define,

$$
\left.|| v\right|_{E}=\sqrt{(v, v)}
$$

for all $v \in V$, the energy norm. A critical relationship between the energy norm and inner-product is called Schwarz' inequality and this inequality

$$
|a(v, w)| \leq|| v||_{E}|| w||_{E} \quad \forall v, w \in V
$$

is a base for Hilbert space theory.

\subsection{Piecewise Polynomial Spaces}

Let $x_{0}=0$ and $x_{n}=1$, so that $x_{0}<x_{1}<\cdots<x_{n}$ and let $S$ is a linear space of functions $v$ such that,

1. $v \in C^{0}[(0,1)]$

2. $\left.v\right|_{x_{i-1}, x_{i}}$ is a linear polynomial, $i=1, \cdots, n$ and

3. $v(0)=0$

\section{Result and Discussion}

\subsection{Finite Element Method Solution}

The steps to determine the finite element solution

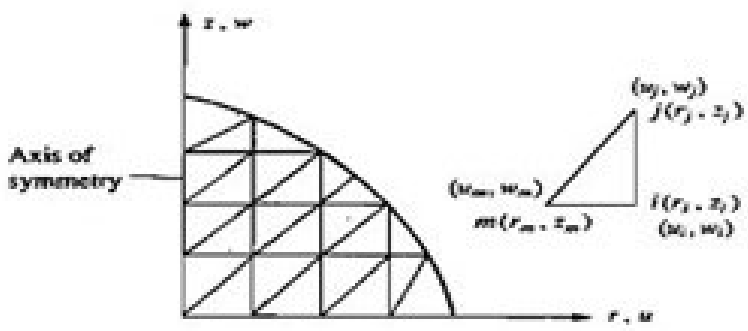

Fig. 1. Discretization axis symmetry into triangular elements.

1. Discretization/meshing and selection of elements.

2. Choose displacement function.

3. Determine equation between stretch relationship $[\xi]$ and deformation $[d]$ as well as between the stretch $[\xi]$ and the stress $[\sigma]$

4. Define the equation matrix and element stiffness. 


$$
\left[\begin{array}{c}
f_{1} \\
f_{2} \\
\vdots \\
f_{n}
\end{array}\right]=\left[\begin{array}{ccccc}
k_{11} & k_{12} & k_{13} & \ldots & k_{1 m} \\
k_{21} & k_{22} & k_{23} & \ldots & k_{2 m} \\
\vdots & \vdots & \vdots & \ddots & \vdots \\
k_{n 1} & k_{n 2} & k_{n 3} & \ldots & k_{n m}
\end{array}\right]\left[\begin{array}{c}
d_{1} \\
d_{2} \\
\vdots \\
d_{n}
\end{array}\right]
$$

or in its simplest form is

$$
[f]=[k][d]
$$

where,

$[f]=$ Force matrix

$[d]=$ Deformation matrix

$[k]=$ Stiffness matrix

\subsection{Finite Element Method with COMSOL Multiphysic 5.2}

COMSOL Multiphysics is a program that uses the concept of finite element method to solve a physical problem and shows simulation of the problem solving. Which basically simulates whatever it wants: simulates heat transfer through complex structures, nanoscale photonic crystals, bending mechanical beams, fluid flow, electrochemical processes, plasma physics and more [5].
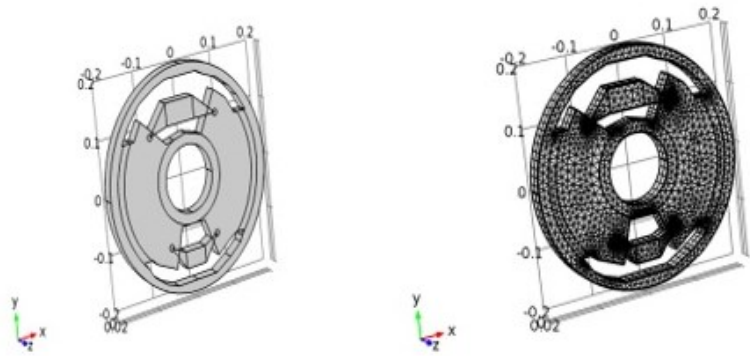

Fig. 2. Brake Model and Mesh with COMSOL.

Parameters used in this research can be seen in Table 1 below.

Table 1. Parameter of brake system

\begin{tabular}{|c|c|c|l|}
\hline Name & Expresion & Value & \multicolumn{1}{|c|}{ Description } \\
\hline$M_{u}$ & 0.3 & 0.3 & Friction Coefficient \\
\hline$v_{0}$ & $25[\mathrm{~m} / \mathrm{s}]$ & $25 \mathrm{~m} / \mathrm{s}$ & Initial Vehicle Speed \\
\hline$a_{0}$ & $-10[\mathrm{~m} / \mathrm{s}]$ & $-10 \mathrm{~m} / \mathrm{s}$ & $\begin{array}{l}\text { Initial } \\
\text { Acceleration }\end{array}$ \\
\hline$r_{\text {wheel }}$ & $0.25[\mathrm{~m}]$ & $0.25 \mathrm{~m}$ & Wheel Radius \\
\hline$m_{\text {bike }}$ & $133[\mathrm{~kg}]$ & $133 \mathrm{~kg}$ & Vehicle Mass \\
\hline tbrakestart & $2[\mathrm{~s}]$ & $2 \mathrm{~s}$ & Braking Time (start) \\
\hline tbrakeend & $4[\mathrm{~s}]$ & $4 \mathrm{~s}$ & Braking Time (end) \\
\hline$T_{\text {air }}$ & $300[\mathrm{~K}]$ & $300 \mathrm{~K}$ & Temperature (air) \\
\hline \multicolumn{3}{|l}{} \\
\multicolumn{2}{|l}{} \\
\hline
\end{tabular}

Based on the function to be used, the speed and acceleration of the movement of the vehicle (in this case is the motorcycle) is presented in the Cartesian graph in Figure 3.

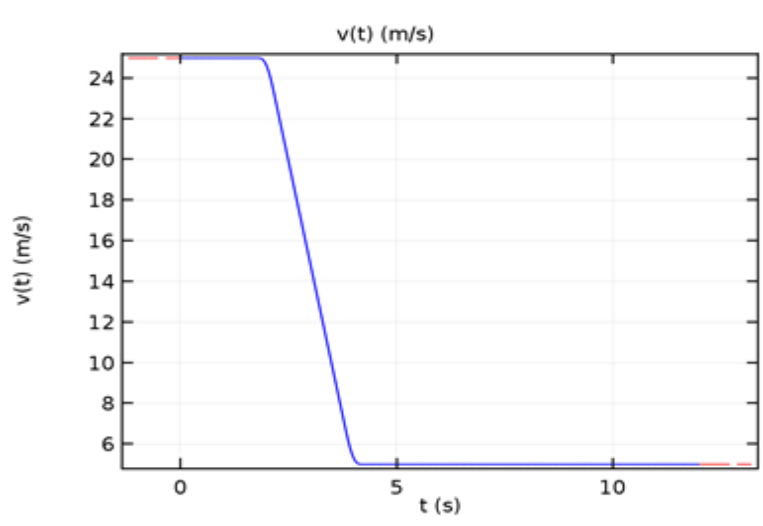

a. Speed

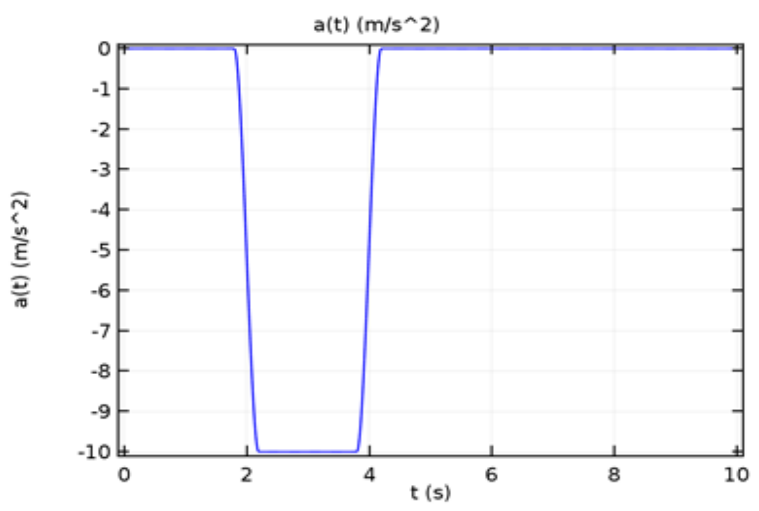

b. Acceleration

Fig. 3. Speed and acceleration graphics on disk

On the contact surface between the brake lining and its tromol, the temperature increases when the brake is used and then decreases again when braking is finish. Visualization results with COMSOL can be seen in the picture below
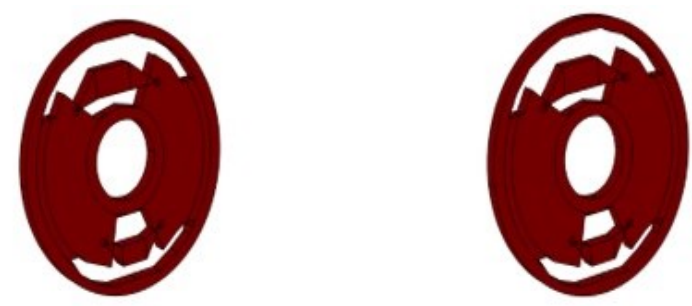

$$
t=0 s
$$$$
t=1.5 \mathrm{~s}
$$
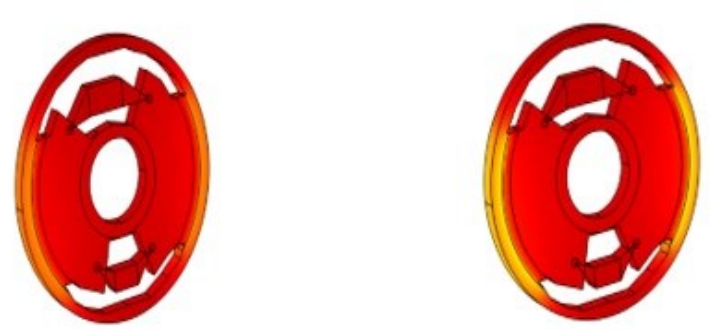

$t=1.95 \mathrm{~s}$ $t=2.6 \mathrm{~s}$

Fig. 4. The surface temperature at the tromol and brake lining at $t=0 \mathrm{~s}$ to $t=2.6 \mathrm{~s}$. 

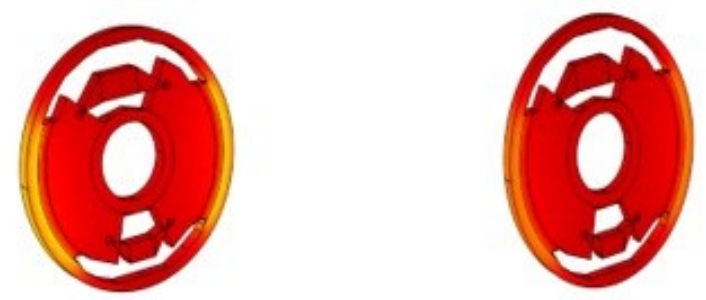

$$
t=2.8 s
$$

$$
t=3.8 s
$$
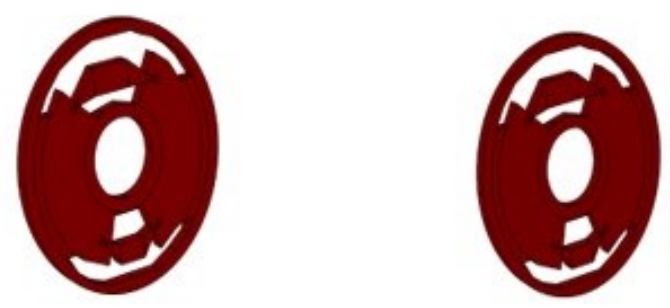

$t=4.6 s$

$t=12 s$
Fig. 5. The surface temperature at the tromol and brake lining at $t=2.8 \mathrm{~s}$ to $t=12 \mathrm{~s}$.

\section{Conclusion}

The heat transfer in the brake lining in this study is one of the problems in heat transfer presented in the form of three dimensions. Where analysis of heat transfer in brake lining based on modelling and visualization results with COMSOL Multiphysic 5.2 software. From the results on modelling and visualization can be seen and analysed heat transfer in the braking process starting from $t=0 \mathrm{~s}$ to $12 \mathrm{~s}$. The results show when braking occurs heat transfer at $t=$ $1.95 \mathrm{~s}$ to $t=3.8 \mathrm{~s}$ and the peak heat friction that occurs can be seen at $t=3.8 \mathrm{~s}$ next temperature will decrease and will return to the initial temperature.

So, it can be concluded that for the allowed length of braking is a maximum of $3.8 \mathrm{~s}$. That is because based on the results of the simulation, if passing time $3.8 \mathrm{~s}$ brake pad will decrease quality (or can be said damaged). So that brake lining will be faster should be replaced if a vehicle often braking more than $3.8 \mathrm{~s}$. That's because the heat transfer past $3.8 \mathrm{~s}$ does not exist anymore or it can be said that excessive heat causes the brake lining to become damaged.

\section{References}

1. Tulus, Ariffin, A.K, Abdullah, S., Muhamad, N. Finite Element Analysis for Heat Transfer in the Insulator on Piston Pin of A Linear Generator Engine. Proceedings of the 2nd IMT-GT Regional Conference Of Mathematics, Statistics And Applications University Sains Malaysia.
2. Marpaung, T.J, Tulus, Suwilo, S. (2018). Cooling Optimization in Tubular Reactor of Palm Oil Waste Processing. Bulletin of Mathematics Vol. 10, No. 01 (2018), pp. 13-24.

3. Hatam, A and Khalkhali,A (2018). Simulation and Sensitivity Analysis of Wear on the Automotive Brake Pad. Simulation Modelling Practice and Theory\} Vol. 84, pp. 106-123.

4. Tulus, Ariffin, A.K, Abdullah, S., Muhamad, N (2008). Heat Transfer Analysis In Magnet Housing Of Linear Generator Using Finite Element Method. Jurnal Kejuruteraan (Journal of Engineering), Vol. 20, pp. 125-133.

5. Tulus, Suriati, and Marpaung T.J. (2018). Sedimentation Optimitation on River Dam Flow by Using COMSOL Multiphysics. Channel IOP Conf. Series: Mater, Sci. Eng $\mathbf{3 0 0} 012051$ 